\title{
Degree Distribution of Arbitrary AANET
}

\author{
Xue Liu, ${ }^{1,2}$ Xiaoping Zeng, ${ }^{1}$ Zhiming Wang, ${ }^{1}$ Li Chen, ${ }^{1}$ and Yuemei Jin ${ }^{1}$ \\ ${ }^{1}$ College of Communication Engineering, Chongqing University, Chongqing 400044, China \\ ${ }^{2}$ Chongqing Communication Institute, Chongqing 400035, China \\ Correspondence should be addressed to Xue Liu; maojiu2005@qq.com
}

Received 26 October 2014; Revised 15 February 2015; Accepted 16 February 2015

Academic Editor: Ronald M. Barrett

Copyright (C) 2015 Xue Liu et al. This is an open access article distributed under the Creative Commons Attribution License, which permits unrestricted use, distribution, and reproduction in any medium, provided the original work is properly cited.

\begin{abstract}
Taking the safe distance between two adjacent planes in the same airline into account, we give a model for the multiairline aeronautical ad hoc network (AANET). Based on our model, we analyze the plane's degree distribution of any arbitrary AANET. Then, the expressions of the degree distributions of one single plane and the whole networks are both worked out and verified by the simulations, in which we generate several random AANETs. Since our model is a reasonable abstraction of the real situation, the theoretical result we get is very close to the result of the real networks, which is also shown in the simulations.
\end{abstract}

\section{Introduction}

With the rapid development of civil aviation, the demands of accessing Internet on board have become stronger than ever before. However, in polar, desert, marine, and other areas where base stations cannot be built, aircrafts have to use satellite links or large span multihop links within AANET to reach base stations. Due to the large delay, high cost, and limited bandwidth of satellite links, AANET is preferred for internet accessing in these regions $[1,2]$.

For AANET, the Newsky project has proposed networking strategies with mobile IPv6 technology [3]. ATENAA project (advanced technologies for networking in aeronautical applications) has studied Ka-band array antennas and optical communications between aircrafts [4]. Rohrer et al. have presented cross-layer networking solutions among physical, mac, network, and transport layers [5]. Vey et al. have proposed the use of direct sequence CDMA (DSCDMA) at the access layer [2] while Yan et al. have studied the capacity of single flight path AANET [6].

In an ad hoc network, the number of a node's neighbors is called "degree" of that node. Thus, the degree distribution of an ad hoc network tells the probability that the degree is an exact number by choosing a node randomly. Based on the degree distribution of the network, a lot of work can be done: the analysis of connectivity and robustness of the network by using the method of generating function formalism [7]; the design of routing and mac protocols; for example, we can choose planes with high degree expectation to relay messages, since usually we cannot know where all the planes are; the site selection of base stations; for example, base station can be set at a place to make sure that the average of the degree expectations of planes in coverage region of that base station is high, which makes it reach more planes through multihop links.

The degree distribution of mobile ad hoc networks has been widely discussed, but most of them are based on the scenarios that nodes in the networks move randomly. However, for AANET and vehicle ad hoc networks (VANET), the nodes are moving along specified lines, which is different from the old scenarios. In VANET, which is also studied a lot in recent years, the speed of vehicles can be affected by plenty of factors (such as pedestrian crosswalks, traffic jams, and traffic lights) and the degree distribution which a vehicle is hard to calculate or even to assume. Thus, the problem about calculating the degree distribution of the networks where nodes move along specified lines has not been solved yet.

In this work, we give a model for the AANET, from which we can approximately calculate the degree distribution of any arbitrary AANET.

\section{The Model}

Since AANET is not deployed currently and none of the achievements mentioned previously is taken as standard or in real use, we do not consider the physical or access layer of 


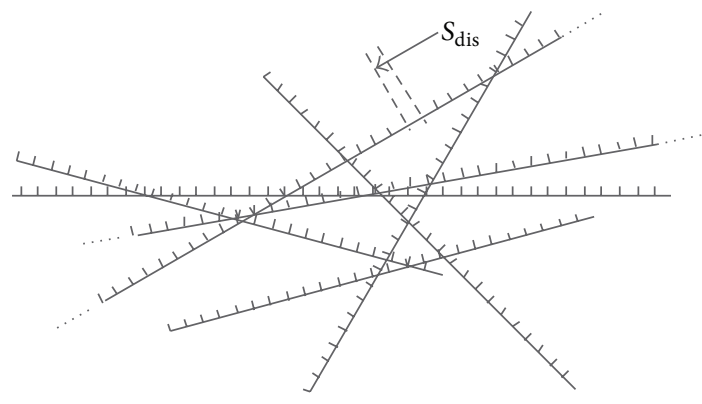

FIgURE 1: The scenario of this work.

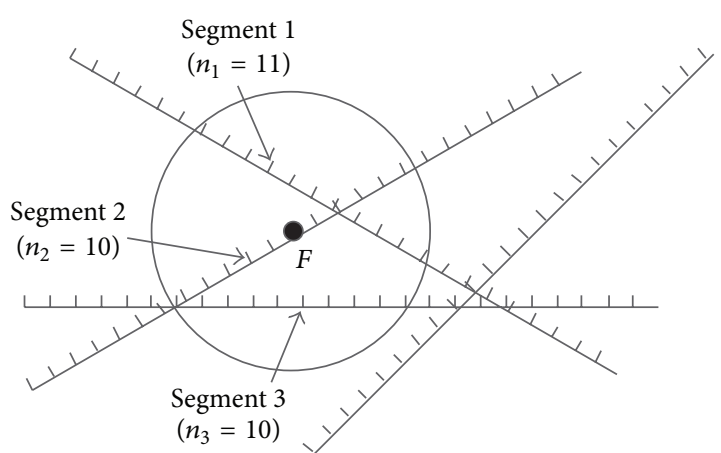

FIGURE 2: The neighbors of a plane.
AANET and use a fixed value, $r$ (between $50 \mathrm{nmi}$ and $300 \mathrm{nmi}$ as used in [8]), as the communication range for all planes.

According to the specifications of the International Civil Aviation Organization (ICAO) [9], a safe distance, call it $S_{\text {dis }}$ (it is around $20 \mathrm{nmi}$ according to ICAO), should be kept between two adjacent planes in the same airline. As the length of an airline is far greater than $S_{\text {dis }}$, we take $S_{\text {dis }}$ as the smallest unit of length. Thus, we assume that the distance between two adjacent planes in the same airline can only be $k S_{\text {dis }}(k=1,2,3, \ldots)$. Since the distance of two airlines at different height level is usually several hundred of meters, which is much less than the assumed planes' communication range $r$, we ignore the distance of two airlines.

In Figure 1, there are several random lines crossing each other with points uniformly spaced on them. The lines stand for airlines while points denote the possible locations of planes. The distance between two adjacent points in the same line is $S_{\text {dis }}$. Furthermore, we assume that the spatial distribution of planes on each airline obeys discrete uniform distribution.

\section{The Degree Distribution of a Plane}

The usual method to calculate the degree distribution of a plane is experimentally based on the scenario that the spatial distribution of planes in each airline follows Poisson point process, and it is easy to tell that the degree distribution of a plane obeys Poisson distribution $[6,10]$. However, the scenario does not take the nonignorable safe distance between planes into account, which is significantly different from the real situation in the sky. For instance, in $[6,10]$, the number of a plane's neighbors can be infinite, but if safe distance is considered, the number of each plane's neighbors has an upper limit.

In Figure 2, there are $m$ airline segments covered by plane $F$ ( $m$ equals 3 in this example). In each segment, there are several possible locations for planes and we denote $n_{i}$ as the number of locations in segment $i(i=1,2, \ldots, m)$, where the location occupied by plane $F$ is not counted. For each location of segment $i$, we denote $P_{i}$ as the probability that a plane is at that location.

Since planes can only be at the designed locations on the airlines, the probability that plane $F$ has $K$ neighbors is the probability that $K$ planes are at the locations covered by plane $F$. Then, using $k_{i}\left(k_{i} \in\left[0, n_{i}\right]\right)$ to denote the number of planes on segment $i$, we can get $K=\sum_{i=1}^{m} k_{i}$ and the probability that there are $k_{i}$ planes on segment $i$ is $C_{n_{i}}^{k_{i}} P_{i}^{k_{i}}\left(1-P_{i}\right)^{n_{i}-k_{i}}$, where $C_{n_{i}}^{k_{i}}$ denotes the number of the ways of setting $k_{i}$ planes at $n_{i}$ locations.

In order to set $K$ planes in total at the locations covered by plane $F$, we set planes following this order: $k_{1}$ to segment 1 , $k_{2}$ to segment 2 , and $k_{m}$ to segment $m$. Thus, the value ranges of $k_{1}$ to $k_{m}$ are as shown in Table 1 .

Therefore, we can obtain the probability that there are $K$ neighbors around plane $F$ according to the order we set planes in

$$
\begin{aligned}
& P(K)=\sum_{k_{1}=0}^{\min \left(n_{1}, K\right)} C_{n_{1}}^{k_{1}} P_{1}^{k_{1}}\left(1-P_{1}\right)^{n_{1}-k_{1}} \\
& \times \sum_{k_{2}=0}^{\min \left(n_{2}, K-k_{1}\right)} C_{n_{2}}^{k_{2}} P_{2}^{k_{2}}\left(1-P_{2}\right)^{n_{2}-k_{2}} \\
& \times \sum_{k_{3}=0}^{\min \left(n_{3}, K-k_{1}-k_{2}\right)} C_{n_{3}}^{k_{3}} P_{3}^{k_{3}}\left(1-P_{3}\right)^{n_{3}-k_{3}} \cdots \\
& \times \quad \sum_{k_{h}=0}^{\min \left(n_{h}, K-\sum_{j=1}^{h-1} k_{j}\right)} C_{n_{h}}^{k_{h}} P_{h}^{k_{h}}\left(1-P_{h}\right)^{n_{h}-k_{h}} \ldots \\
& \times \quad \sum_{k_{m-1}=0}^{\min \left(n_{m-1}, K-\sum_{j=1}^{m-2} k_{j}\right)} C_{n_{m-1}}^{k_{m-1}} P_{m-1}^{k_{m-1}}\left(1-P_{m-1}\right)^{n_{m-1}-k_{m-1}} G, \\
& K \in\left[0, \sum_{i=1}^{m} n_{i}\right]
\end{aligned}
$$

$G= \begin{cases}0, & \text { others } \\ C_{n_{m}}^{H} P_{m}^{H}\left(1-P_{m}\right)^{n_{m}-H}, & n_{m} \geq H=K-\sum_{j=1}^{m-1} k_{j} \geq 0 .\end{cases}$ 
TABLE 1: Value range for $k_{i}$.

\begin{tabular}{lc}
\hline$k_{i}$ & Value range \\
\hline$k_{1}$ & {$\left[0, \min \left(n_{1}, K\right)\right]$} \\
$k_{2}$ & {$\left[0, \min \left(n_{2}, K-k_{1}\right)\right]$} \\
$k_{3}$ & {$\left[0, \min \left(n_{3}, K-k_{1}-k_{2}\right)\right]$} \\
$\vdots$ & $\vdots$ \\
$k_{h}$ & {$\left[0, \min \left(n_{h}, K-\sum_{j=1}^{h-1} k_{j}\right)\right]$} \\
$\vdots$ & $\vdots$ \\
$k_{m}$ & $K-\sum_{j=1}^{m-1} k_{j}$ \\
\hline
\end{tabular}

Denoting $f_{i}\left(k_{i}\right)$ as $C_{n_{i}}^{k_{i}} P_{i}^{k_{i}}\left(1-P_{i}\right)^{n_{i}-k_{i}}$, we can get a simpler expression for $P(K)$ in

$$
\begin{gathered}
P(K)=\sum_{k_{1}=0}^{\min \left(n_{1}, K\right)} \sum_{k_{2}=0}^{\min \left(n_{2}, K-k_{1}\right)} \sum_{k_{3}=0}^{\min \left(n_{3}, K-k_{1}-k_{2}\right)} \cdots \sum_{k_{h}=0}^{\min \left(n_{h}, K-\sum_{j=1}^{h-1} k_{j}\right)} \\
\ldots \sum_{k_{m-1}=0}^{\min \left(n_{m-1}, K-\sum_{j=1}^{m-2} k_{j}\right)}\left(\left[f_{m}\left(k_{m}\right)\right]_{n_{m} \geq k_{m} \geq 0} \prod_{i=1}^{m-1} f_{i}\left(k_{i}\right)\right), \\
{\left[f_{m}\left(k_{m}\right)\right]_{n_{m} \geq k_{m} \geq 0}=\left\{\begin{array}{l}
\left.0, \sum_{i=1}^{m} n_{i}\right], \\
\text { others } \\
f_{m}\left(k_{m}\right), \quad n_{m} \geq k_{m}=K-\sum_{j=1}^{m-1} k_{j} \geq 0 .
\end{array}\right.}
\end{gathered}
$$

\section{The Degree Distribution of AANET}

Assume $M$ airlines which are in Figure 1. Each airline has $N_{i}(i=1,2 \ldots, M)$ locations for planes. $P_{i}$ denotes the probability that a plane is a location of airline $i$. For plane on each location in the networks, we can calculate its degree distribution by using the method in Section 3. Then, we denote $P_{i j}(K)\left(i \in[0, M], j \in\left[0, N_{i}\right]\right)$ as the probability that a plane at the $j$ th location of airline $i$ has $K$ neighbors and uses $K_{i j}$ to represent the maximum number of neighbors of the plane on that location. Thus, to calculate the probability that a plane in the AANET has $K$ neighbors, we add all the $P_{i j}(K)$ with multiplying plane's existing probability at the $j$ th location of airline $i$, which is $P_{i} / \sum_{i}^{M} N_{i} P_{i}$. Then, we obtain the degree distribution of the AANET in

$$
P(K)=\frac{\sum_{i=1}^{M} \sum_{j=1}^{N_{i}} P_{i}\left[P_{i j}(K)\right]_{K \leq K_{i j}}}{\sum_{i=1}^{M} N_{i} P_{i}} .
$$

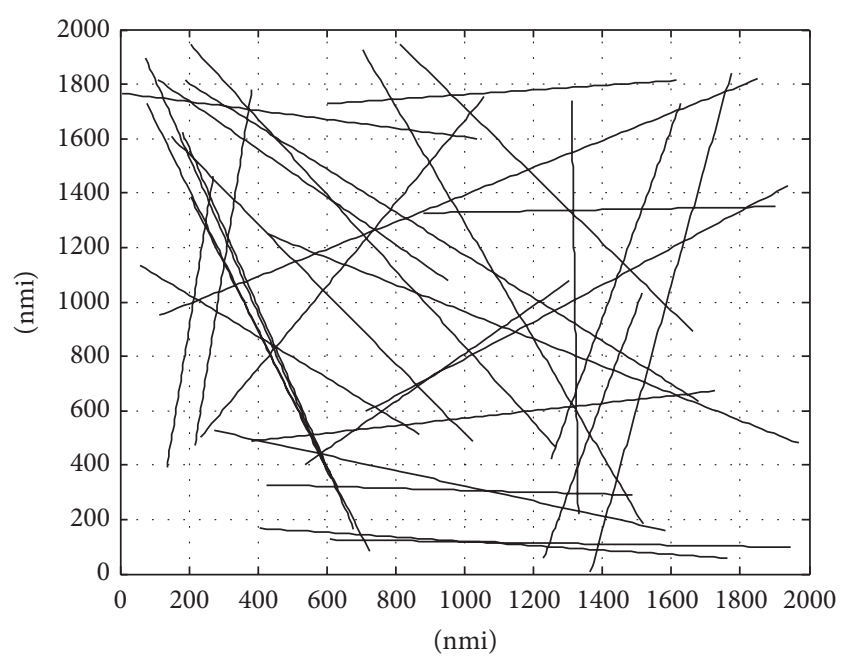

Figure 3: Scenario 1 (30 airlines).

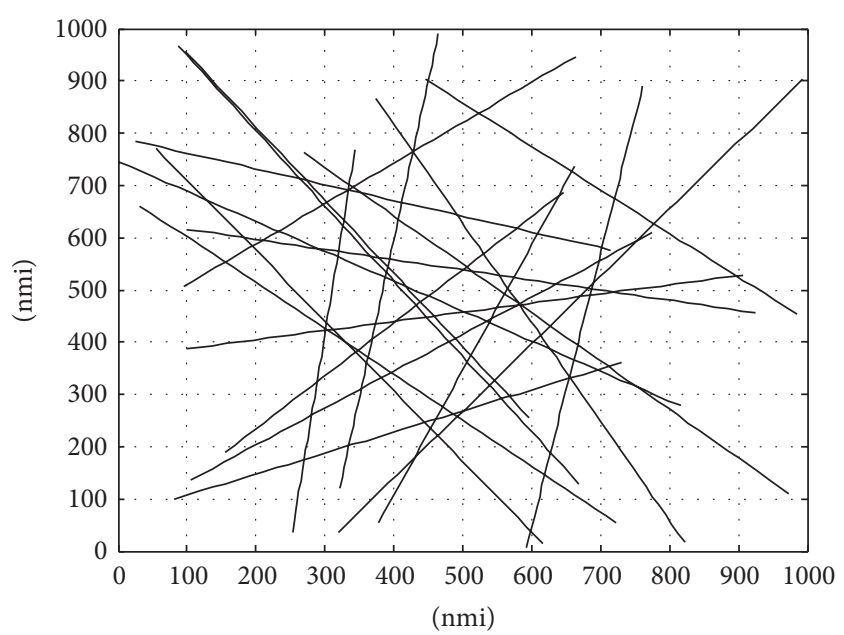

FIGURE 4: Scenario 2 (20 airlines).

\section{Numeric Results}

For verifying our work, we generate three random scenarios and draw them in Figures 3 to 5, respectively, whose detailed parameters are listed in the appendix. In each scenario, we use MATLAB to generate plane for each location in airline $i$ with probability $P_{i}$ (from 0.5 to 1 , see the appendix). After the generation, we record the number of the planes and every plane's neighbors (degree). Then, we delete all the planes in the scenario and generate planes again in the same way. We repeatedly generate planes in one scenario for 100000 times. Then, by using $N$ to denote the total number of planes generated in 100000 times and $m_{i}(i=1,2,3, \ldots)$ to denote the number of planes with degree $i$, we can get the approximate degree distribution of that scenario through

$$
P(K) \approx \frac{m_{K}}{N} .
$$

We calculate the theoretical value in (5) and simulation value in (6) for each scenario in Figures 3 to 5 and draw their 


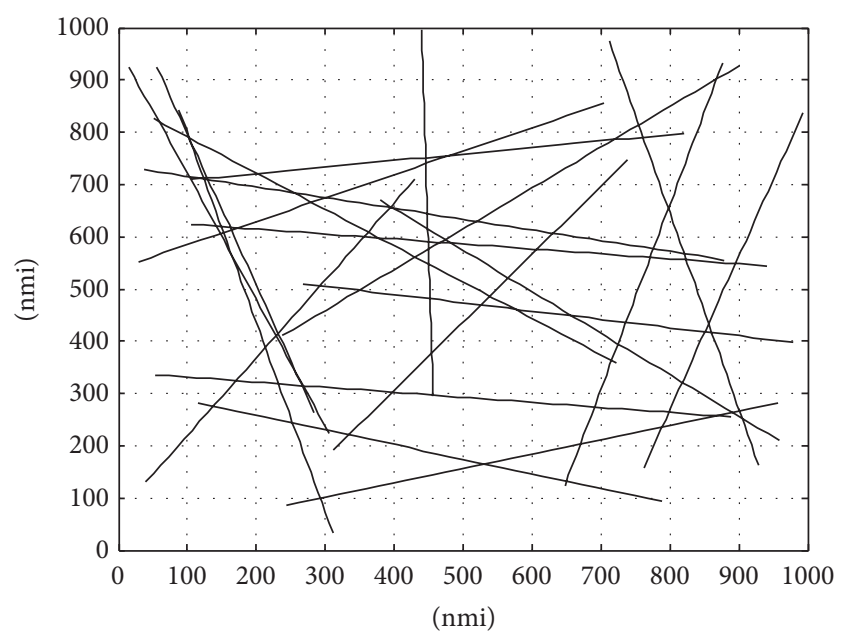

FIGURE 5: Scenario 3 (20 airlines).

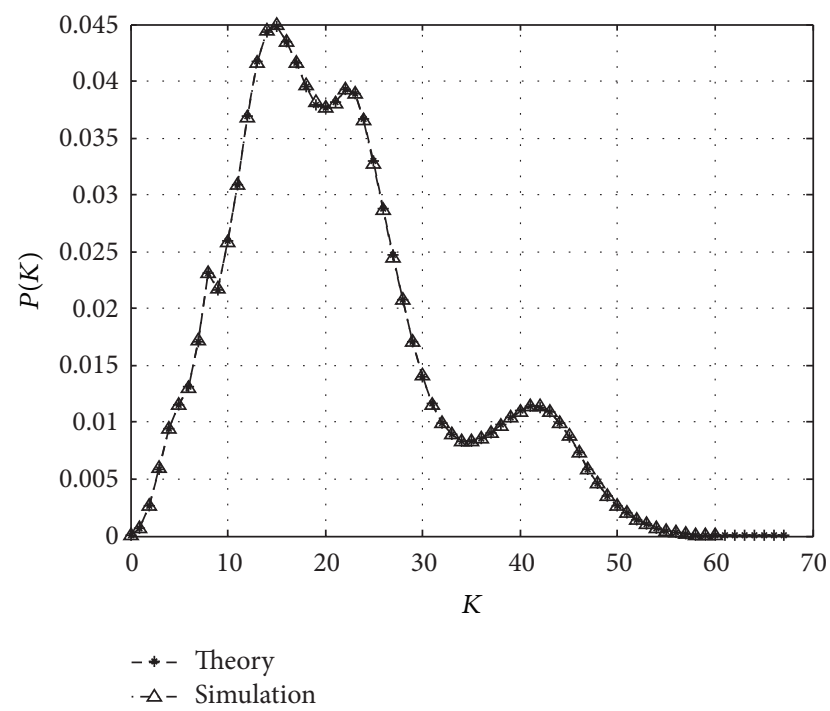

FIGURE 6: Results for scenario $1\left(r=100 \mathrm{nmi}, S_{\text {dis }}=20 \mathrm{nmi}\right)$.

results in Figures 6 to 8, respectively, where the theory and simulation lines are overlapped.

From Figures 6 to 8, we can see that the probability that a plane has no neighbor is nearly zero, because a plane at most of the locations (except the locations near the endpoints of the airlines) can cover at least 6 locations in its own airline in scenarios 2 and $3\left(r=70 \mathrm{nmi}, S_{\text {dis }}=20 \mathrm{nmi}\right)$ and 10 locations in scenario $1\left(r=100 \mathrm{nmi}, S_{\mathrm{dis}}=20 \mathrm{nmi}\right)$. With considering that every $P_{i}$ we choose is larger than 0.5 , the probability that a plane has no neighbor should certainly be very small. Another thing we can tell from the figures is that the probability decays to zero for large degrees. As we inform in Section 3, the number of each plane's neighbors has an upper limit since a safe distance should be kept between two planes. When a plane's degree reaches the upper limit, this means that every location covered by that plane should have a plane on it. Thus, when the upper limit is big, the probability

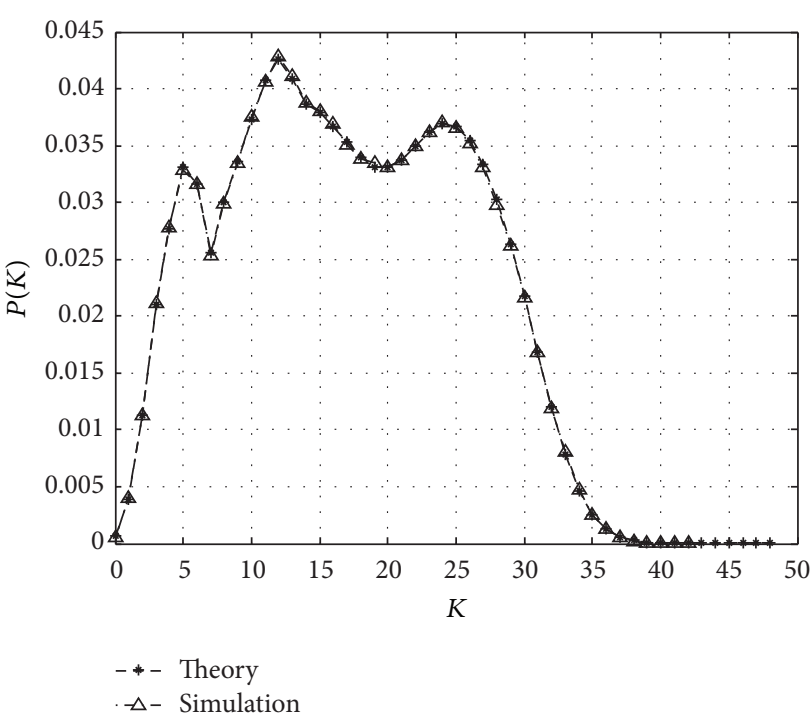

FIgURE 7: Results for scenario $2\left(r=70 \mathrm{nmi}, S_{\text {dis }}=20 \mathrm{nmi}\right)$.

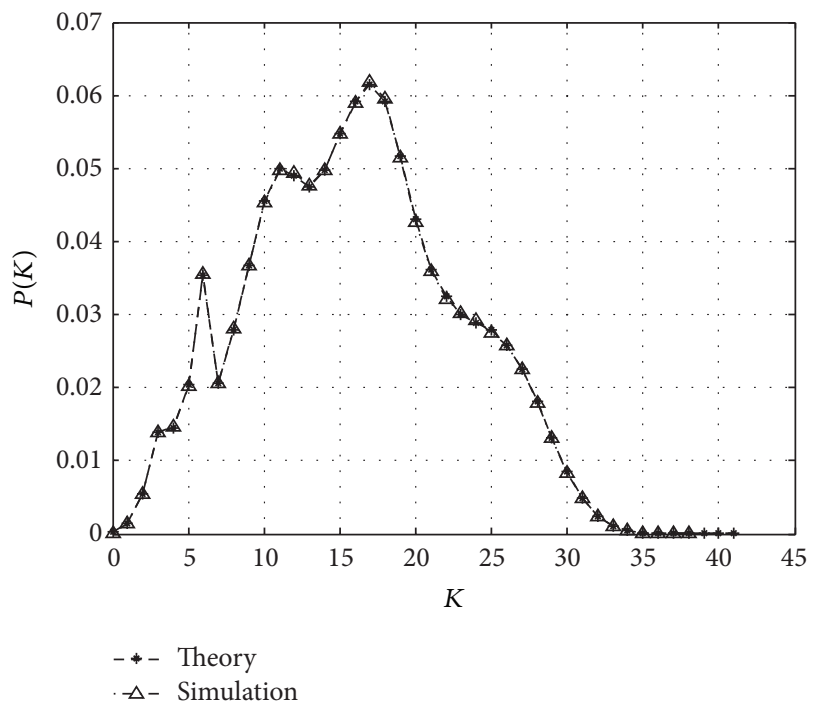

FIgURE 8: Results for scenario 3 ( $\left.r=70 \mathrm{nmi}, S_{\text {dis }}=20 \mathrm{nmi}\right)$.

of reaching that limit should be very small and, for the degrees beyond the maximum upper limit, it should be zero.

The peaks of Figures 7 to 9 are related mostly on the communication range and safe distance, because those two parameters decide how many locations a plane can cover; for example, plane at most of the locations (more than 50\%) in scenario 1 can cover over 15 to 20 locations (counted by computer), so the peak of scenario 1 should most likely be around 15 and 20, and Figure 6 proves that, for scenarios 2 and 3 , they are 10 to 20 and 15 to 22 , respectively.

Since, in the real world, planes cannot be exactly at the locations we draw in our scenarios, we give a random shift for every plane after each generation of planes. Then, we run the simulation again to see the deviation between our theoretical model and the real situation. The results are shown 


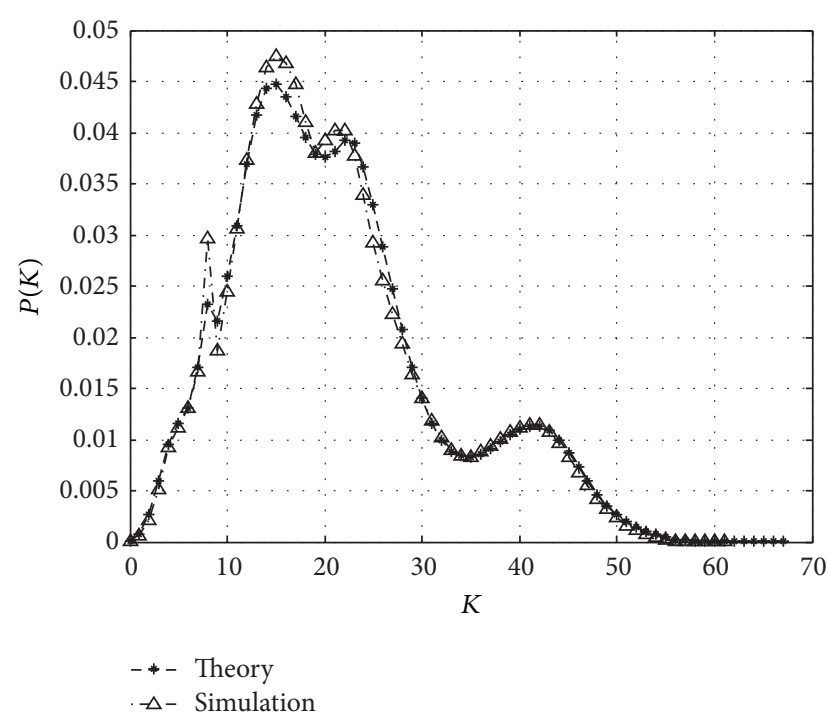

FIGURE 9: Results for scenario 1 with random shift $(r=$ $\left.100 \mathrm{nmi}, S_{\text {dis }}=20 \mathrm{nmi}\right)$.

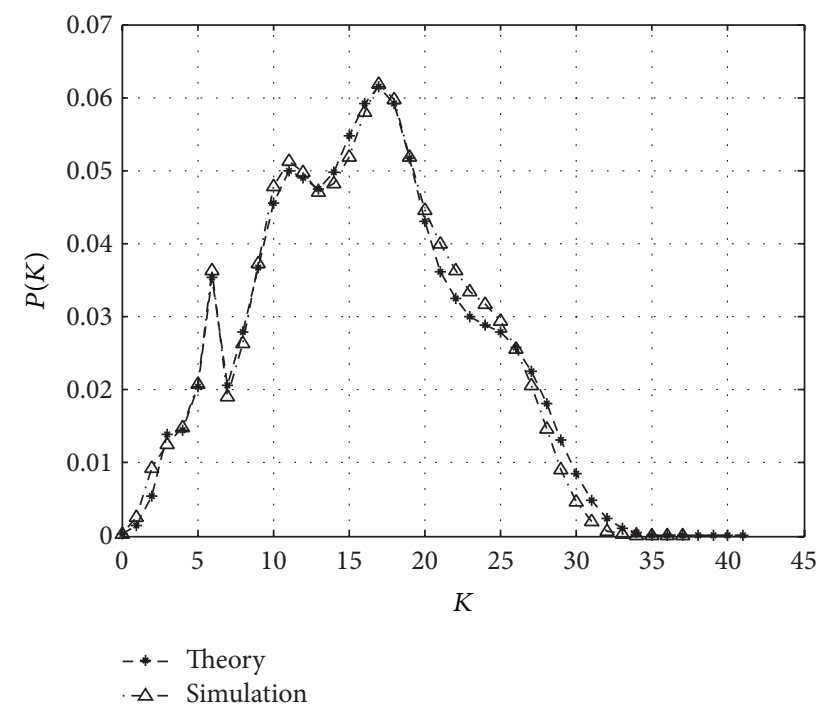

FIgURE 10: Results for scenario 3 with random shift $(r=$ $70 \mathrm{nmi}, S_{\text {dis }}=20 \mathrm{nmi}$ ).

in Figures 9 to 11 and the random shift strategy is described in the following paragraph.

For each airline, we move its first plane (left most plane) a distance of $L_{1}$ along the airline, where $L_{1}$ is uniformly distributed between $-S_{\text {dis }} / 2$ and $S_{\text {dis }} / 2$. For the second plane, we move it a distance of $L_{2}$. $L_{2}$ has two different distributions according to whether it is at the location adjacent to the first plane's or not. If yes, $L_{2}$ is uniformly distributed between $L_{1}$ and $S_{\text {dis }} / 2$ to make sure the distance of the two planes is not less than $S_{\text {dis }}$. If no, $L_{2}$ has the same distribution of $L_{1}$. Following this way, we move the $j$ th plane according

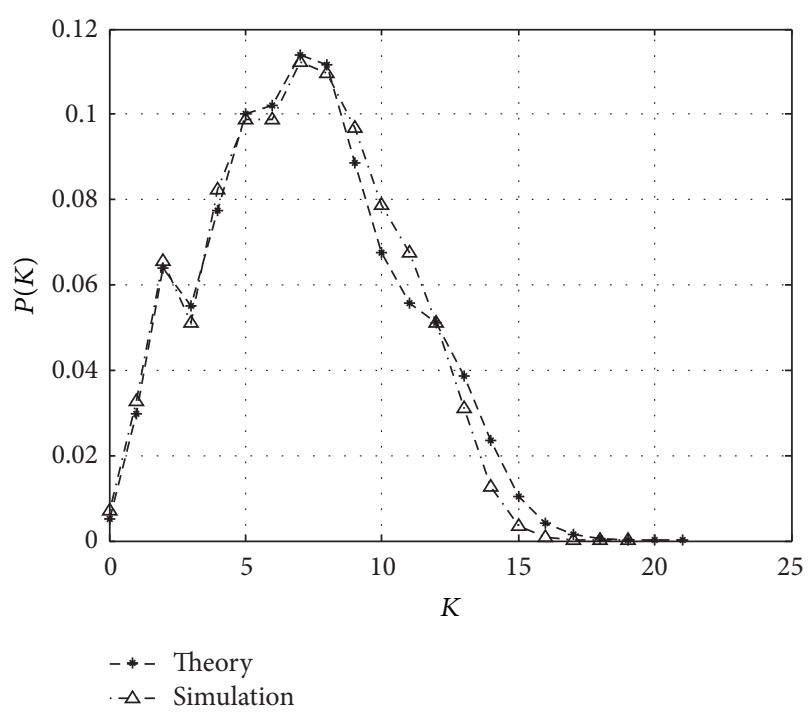

FIGURE 11: Results for scenario 3 with random shift $(r=$ $\left.70 \mathrm{nmi}, S_{\text {dis }}=40 \mathrm{nmi}\right)$.

to whether the $(j-1)$ th and $j$ th planes are at the adjacent locations on that airline.

From Figures 9 to 11, we can see there is only a small deviation between our model and the real situation, and it is believed to be decreasing with the ratio of $r$ to $S_{\text {dis }}$. In Figures 10 and 11, we use the same scenario with different safe distances. The shapes of the two figures are similar, but the mathematical expectation of $K$ in Figure 10 is nearly the double of that in Figure 11. This is because scenario can form the shape, while safe distance can decide the plane density.

\section{Conclusion}

In this paper, we introduce a model of AANET, which considers the nonignorable safe distance between planes. Based on this model, the plane's degree distribution of an arbitrary AANET is analyzed. We find that, different from other works, the degree of each plane in our model has an upper limit, which is coursed by the safe distance. Then, we get from the numeric results of our theoretical method that the peaks of the degree distribution are related mostly on the communication range and safe distance, and the probability of getting large values of degree is very small and it is zero for the degrees beyond the maximum upper limit.

For future research, we will consider simulating a network around some real-world flight paths as opposed to changing the distribution of random paths to show the utility of our method in a real-world case.

\section{Appendix}

See Tables 2, 3, and 4 . 
TABLE 2: Parameters of scenario 1.

\begin{tabular}{lccc}
\hline & Airline & Range of $x$ & $P$ \\
\hline 1 & $2.58 x+y-1929.54=0$ & {$[77.95,613.29]$} & 0.6 \\
2 & $0.76 x+y-1178.04=0$ & {$[58.39,879.89]$} & 0.89 \\
3 & $0.02 x+y-139.37=0$ & {$[608.17,1953.46]$} & 0.89 \\
4 & $0.04 x+y-342.61=0$ & {$[427.14,1489.82]$} & 0.82 \\
5 & $-4.49 x+y+6127.43=0$ & {$[1364.92,1776.88]$} & 0.95 \\
6 & $0.50 x+y-1461.92=0$ & {$[429.48,1979.71]$} & 0.75 \\
7 & $-0.88 x+y+69.05=0$ & {$[536.53,1311.26]$} & 0.72 \\
8 & $-1.52 x+y-146.88=0$ & {$[233.33,1059.16]$} & 0.53 \\
9 & $-7.97 x+y+692.72=0$ & {$[135.93,270.73]$} & 0.65 \\
10 & $1.24 x+y-2958.86=0$ & {$[811.29,1665.92]$} & 0.73 \\
11 & $-3.49 x+y+3939.50=0$ & {$[1251.73,1628.00]$} & 0.55 \\
12 & $0.16 x+y-1768.40=0$ & {$[7.73,1035.99]$} & 0.74 \\
13 & $0.79 x+y-1962.16=0$ & {$[187.84,1679.15]$} & 0.69 \\
14 & $-3.42 x+y+4146.90=0$ & {$[1229.30,1520.74]$} & 0.52 \\
15 & $-0.02 x+y-1306.39=0$ & {$[880.62,1911.55]$} & 0.87 \\
16 & $0.08 x+y-200.58=0$ & {$[405.60,1777.07]$} & 0.64 \\
17 & $2.50 x+y-1897.33=0$ & {$[204.49,731.02]$} & 0.77 \\
18 & $2.93 x+y-2154.74=0$ & {$[180.99,683.54]$} & 0.75 \\
19 & $0.88 x+y-1912.86=0$ & {$[109.41,953.64]$} & 0.57 \\
20 & $1.39 x+y-2231.38=0$ & {$[203.71,1264.09]$} & 0.55 \\
21 & $79.68 x+y-106171.04=0$ & {$[1310.57,1329.72]$} & 0.51 \\
22 & $1.28 x+y-1800.32=0$ & {$[151.01,1028.81]$} & 0.77 \\
23 & $-7.95 x+y+1258.15=0$ & {$[217.53,383.32]$} & 0.53 \\
24 & $2.14 x+y-3430.21=0$ & {$[703.89,1519.09]$} & 0.67 \\
25 & $0.28 x+y-603.50=0$ & {$[274.77,1588.72]$} & 0.74 \\
26 & $-0.09 x+y-1677.97=0$ & {$[599.14,1635.26]$} & 0.55 \\
27 & $-0.14 x+y-435.09=0$ & {$[379.64,1737.19]$} & 0.83 \\
\hline & $-0.50 x+y-892.90=0$ & {$[113.78,1862.92]$} & 0.87 \\
$29.88 x+y-2110.97=0$ & {$[74.49,603.50]$} & 0.96 \\
& $-0.68 x+y-111.87=0$ & {$[713.92,1950.68]$} & 0.95 \\
\hline
\end{tabular}

\section{Conflict of Interests}

The authors declare that there is no conflict of interests regarding the publication of this paper.

\section{Acknowledgments}

This research is supported by the National Natural Science Foundation of China (Grants nos. 61171089, 91438104, and 61302054) and Chongqing Science \& Technology Commission (Grants nos. cstc2014yykfA40002 and cstc2012jjb40010).
TABle 3: Parameters of scenario 2.

\begin{tabular}{lccc}
\hline & Airline & Range of $x$ & $P$ \\
\hline 1 & $1.35 x+y-844.70=0$ & {$[55.61,620.46]$} & 0.53 \\
2 & $0.30 x+y-792.12=0$ & {$[25.45,725.70]$} & 0.66 \\
3 & $-0.40 x+y-66.80=0$ & {$[80.66,747.52]$} & 0.73 \\
4 & $-5.24 x+y+3096.09=0$ & {$[592.50,761.51]$} & 0.73 \\
5 & $0.93 x+y-1012.40=0$ & {$[269.63,980.69]$} & 0.81 \\
6 & $-6.06 x+y+1825.23=0$ & {$[321.40,464.85]$} & 0.69 \\
7 & $0.19 x+y-635.55=0$ & {$[99.68,928.24]$} & 0.6 \\
8 & $-8.03 x+y+1991.97=0$ & {$[252.56,346.31]$} & 0.53 \\
9 & $-1.29 x+y+375.77=0$ & {$[319.11,998.05]$} & 0.82 \\
10 & $-1.01 x+y-31.62=0$ & {$[155.09,650.33]$} & 0.91 \\
11 & $1.90 x+y-1575.72=0$ & {$[374.37,826.71]$} & 0.55 \\
12 & $-0.18 x+y-369.57=0$ & {$[98.74,915.06]$} & 0.56 \\
13 & $0.57 x+y-745.92=0$ & {$[0.62,816.80]$} & 0.66 \\
14 & $-0.71 x+y-61.87=0$ & {$[104.25,782.36]$} & 0.81 \\
15 & $-2.39 x+y+847.86=0$ & {$[377.03,665.98]$} & 0.71 \\
16 & $0.88 x+y-688.99=0$ & {$[31.63,723.34]$} & 0.79 \\
17 & $-0.78 x+y-433.04=0$ & {$[94.40,673.71]$} & 0.84 \\
18 & $1.44 x+y-1092.75=0$ & {$[86.99,676.56]$} & 0.83 \\
19 & $0.83 x+y-1274.48=0$ & {$[446.49,991.50]$} & 0.73 \\
20 & $1.40 x+y-1092.81=0$ & {$[97.50,597.67]$} & 0.91 \\
\hline
\end{tabular}

TABLE 4: Parameters of scenario 3.

\begin{tabular}{cccc}
\hline & Airline & Range of $x$ & $P$ \\
\hline 1 & $2.89 x+y-1085.85=0$ & {$[55.84,286.33]$} & 0.86 \\
2 & $0.09 x+y-633.54=0$ & {$[104.28,954.11]$} & 0.71 \\
3 & $-1.30 x+y+213.65=0$ & {$[311.92,743.70]$} & 0.57 \\
4 & $0.79 x+y-971.51=0$ & {$[378.87,967.53]$} & 0.88 \\
5 & $-0.78 x+y-224.71=0$ & {$[237.94,912.15]$} & 0.74 \\
6 & $-2.94 x+y+2079.82=0$ & {$[761.69,998.72]$} & 0.98 \\
7 & $0.16 x+y-552.34=0$ & {$[268.09,986.72]$} & 0.94 \\
8 & $0.20 x+y-736.46=0$ & {$[37.21,896.98]$} & 0.58 \\
9 & $-0.13 x+y-696.10=0$ & {$[105.55,824.21]$} & 0.91 \\
10 & $2.41 x+y-961.32=0$ & {$[14.72,311.12]$} & 0.83 \\
11 & $0.10 x+y-340.86=0$ & {$[53.07,905.06]$} & 0.94 \\
12 & $3.63 x+y-1161.00=0$ & {$[87.59,315.56]$} & 0.9 \\
13 & $-3.54 x+y+2166.35=0$ & {$[647.85,880.35]$} & 0.75 \\
14 & $-0.45 x+y-539.45=0$ & {$[28.99,709.69]$} & 0.83 \\
15 & $0.28 x+y-313.95=0$ & {$[114.26,795.89]$} & 0.77 \\
16 & $-0.28 x+y-17.43=0$ & {$[243.10,971.41]$} & 0.87 \\
17 & $3.75 x+y-3643.45=0$ & {$[711.96,929.24]$} & 0.82 \\
18 & $-1.49 x+y-71.08=0$ & {$[39.60,432.18]$} & 0.87 \\
19 & $0.69 x+y-860.79=0$ & {$[50.20,724.29]$} & 0.76 \\
20 & $41.71 x+y-19333.33=0$ & {$[439.63,456.41]$} & 0.55 \\
\hline & & &
\end{tabular}




\section{References}

[1] E. Sakhaee and A. Jamalipour, "The global in-flight internet," IEEE Journal on Selected Areas in Communications, vol. 24, no. 9, pp. 1748-1757, 2006.

[2] Q. Vey, A. Pirovano, J. Radzik, and F. Garcia, "Aeronautical Ad Hoc network for civil aviation," in Communication Technologies for Vehicles, vol. 8435 of Lecture Notes in Computer Science, pp. 81-93, Springer International Publishing, 2014.

[3] M. Schnell and S. Scalise, "NEWSKY - concept for NEtworking the SKY for civil aeronautical communications," IEEE Aerospace and Electronic Systems Magazine, vol. 22, no. 5, pp. 25-29, 2007.

[4] K. Karras, T. Kyritsis, M. Amirfeiz, and S. Baiotti, "Aeronautical mobile ad hoc networks," in Proceedings of the 14th European Wireless Conference, pp. 1-6, June 2008.

[5] J. P. Rohrer, A. Jabbar, E. K. Cetinkaya, E. Perrins, and J. P. G. Sterbenz, "Highly-dynamic cross-layered aeronautical network architecture," IEEE Transactions on Aerospace and Electronic Systems, vol. 47, no. 4, pp. 2742-2765, 2011.

[6] J. Yan, C. Hua, C. Chen, and X. Guan, "The capacity of aeronautical ad-hoc networks," Wireless Networks, vol. 20, no. 7, pp. 2123-2130, 2014.

[7] R. Albert and A.-L. Barabási, "Statistical mechanics of complex networks," Reviews of Modern Physics, vol. 74, article 47, 2002.

[8] D. Medina, F. Hoffmann, S. Ayaz, and C.-H. Rokitansky, "Feasibility of an aeronautical mobile ad hoc network over the north atlantic corridor," in Proceedings of the 5th Annual IEEE Communications Society Conference on Sensor, Mesh and Ad Hoc Communications and Networks, pp. 109-116, usa, June 2008.

[9] The Convention of International Civil Aviation Organization, Annex 2, 'rules of air', 2012.

[10] B. Zheng, H.-Y. Zhang, P. Sun, and G.-C. Huang, "Connectivity on one- and two-way flight routes in aeronautical Ad hoc networks," Journal of Shanghai Jiaotong University, vol. 46, no. 4, pp. 624-629, 2012. 

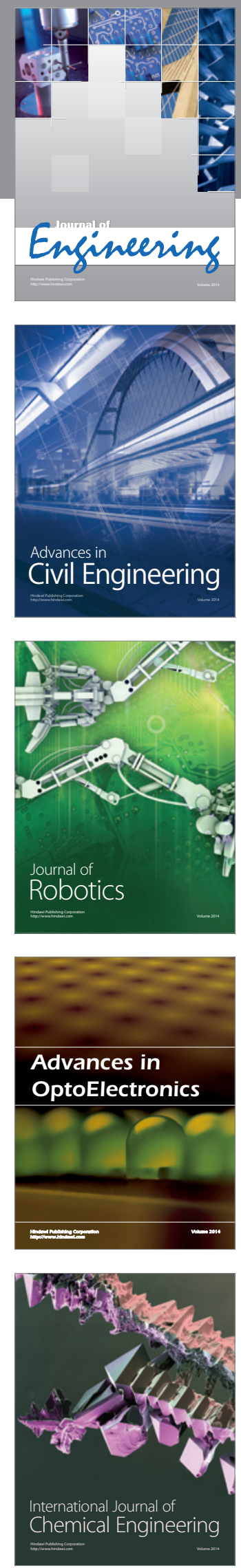

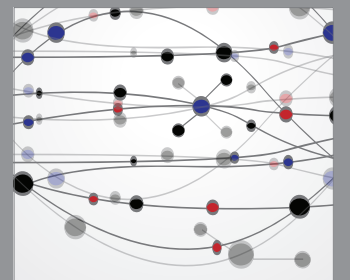

The Scientific World Journal
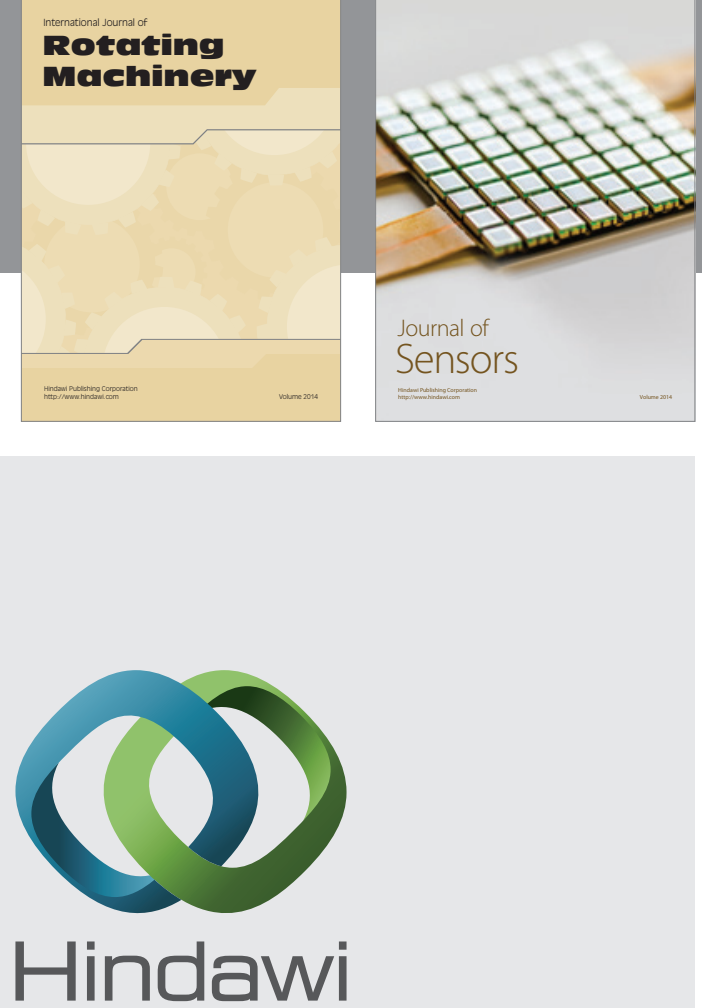

Submit your manuscripts at http://www.hindawi.com
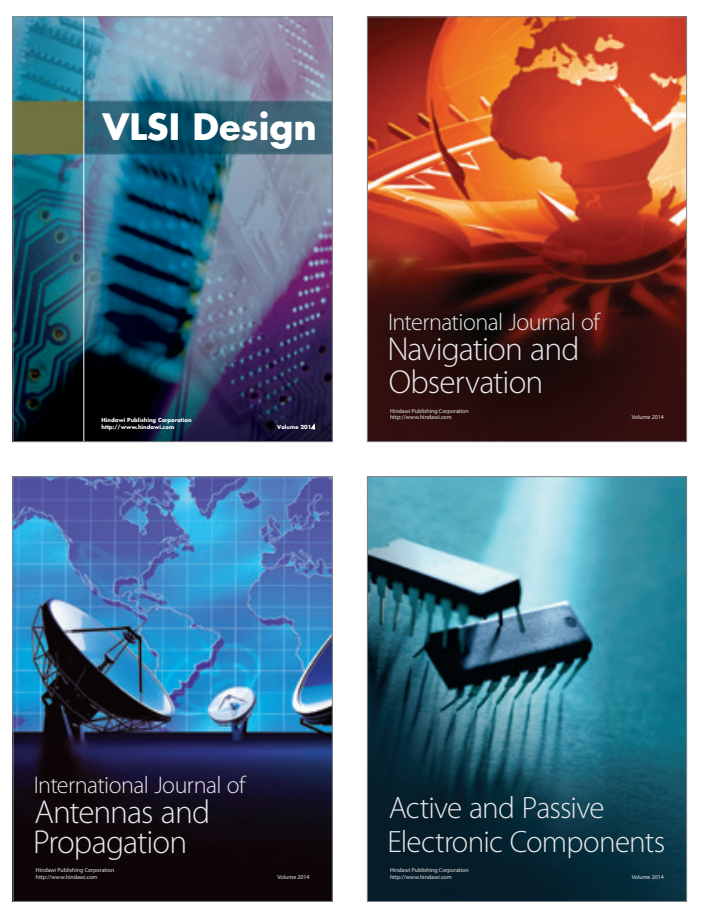
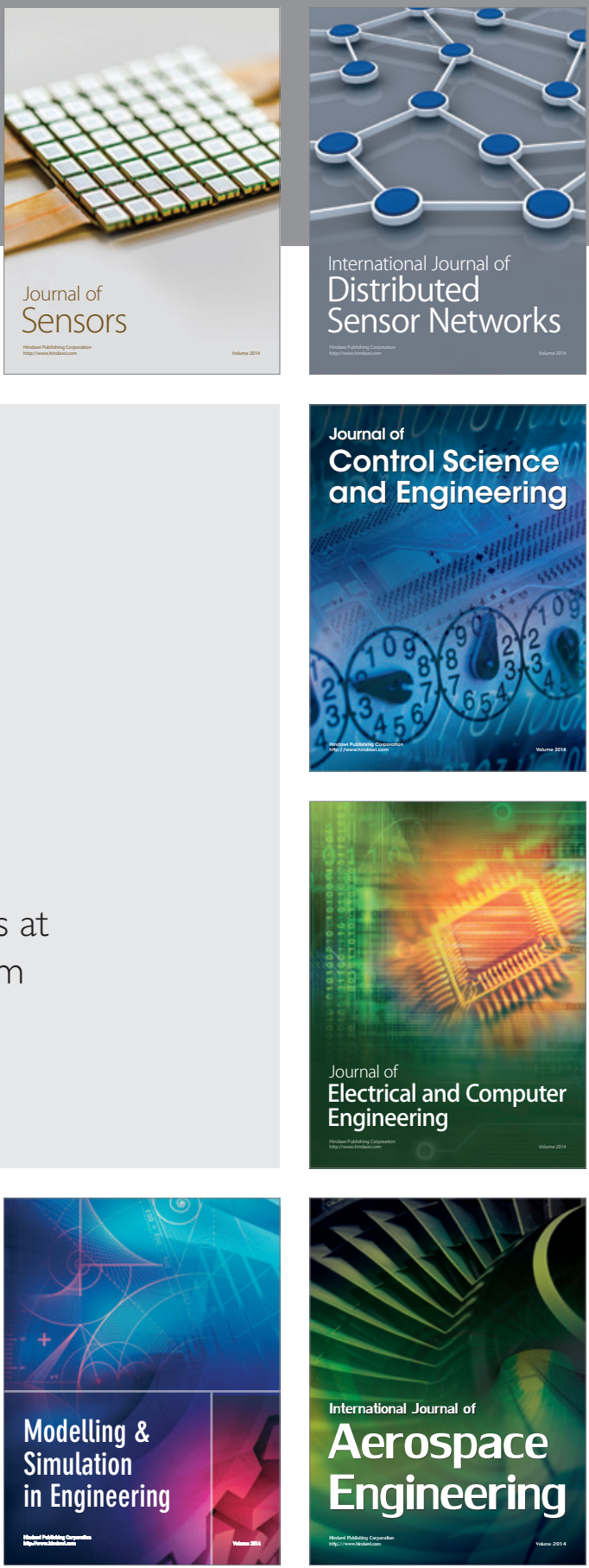

Journal of

Control Science

and Engineering
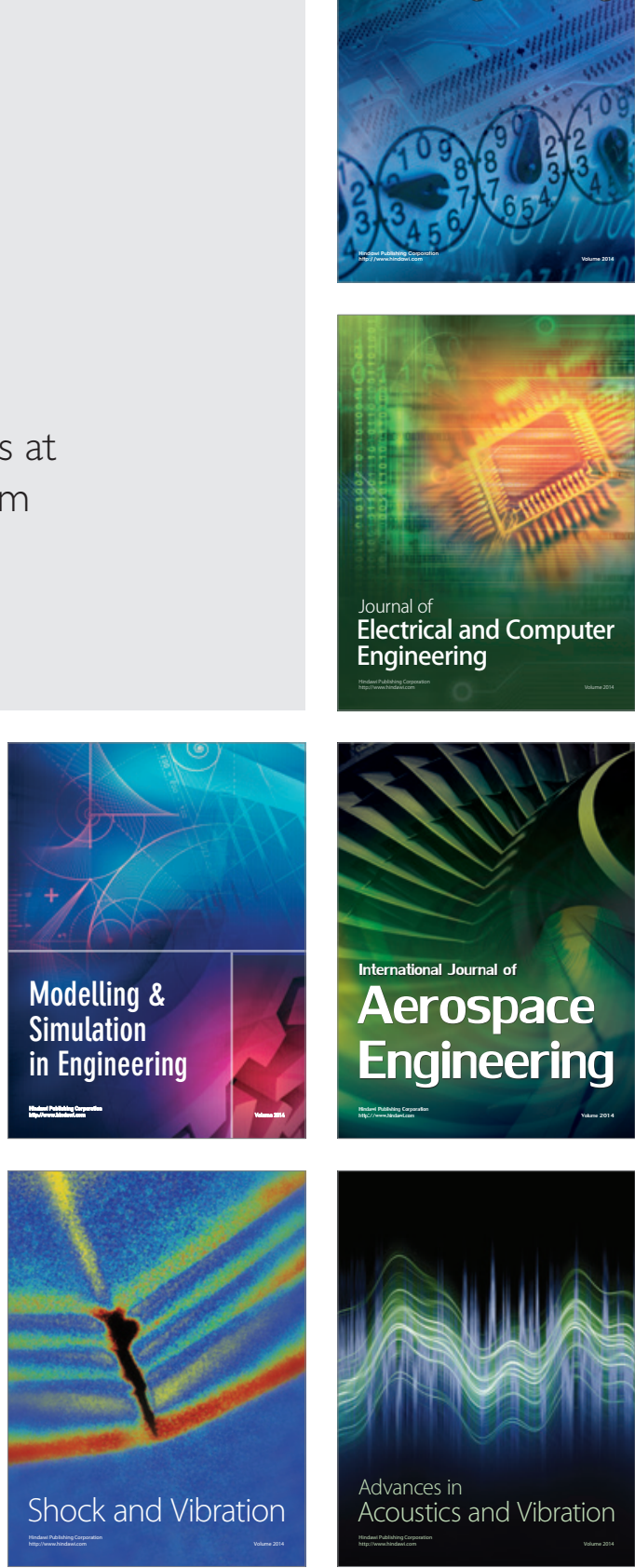